QUARTERLY OF APPLIED MATHEMATICS

VOLUME LXX, NUMBER 1

MARCH 2012, PAGES 181-198

S $0033-569 X(2011) 01252-2$

Article electronically published on September 19, 2011

\title{
SLOW DECAY FOR A LINEARIZED MODEL OF THE SOLAR WIND
}

\author{
BY \\ JACK SCHAEFFER \\ Department of Mathematical Sciences, Carnegie Mellon University, Pittsburgh, Pennsylvania 15213
}

\begin{abstract}
The solar wind interacting with a magnetized obstacle is modelled with the steady Vlasov-Poisson system in the plane. The system is linearized for the (given) magnetic field of the obstacle being small. The main focus is on the rate of decay of the spatial charge density "downwind" of the obstacle. A special case that admits an explicit solution is presented. It is also shown that when the background particle distribution is compactly supported in velocity, that the spatial charge density cannot, in general, decay faster than $x_{1}^{-\frac{1}{2}}$, where $x_{1}$ is the downwind distance.
\end{abstract}

1. Introduction. Consider the following simplified model of the steady solar wind:

$$
\left\{\begin{array}{l}
v \cdot \nabla_{x} f-\left(E_{1}+v_{2} B\right) \partial_{v_{1}} f-\left(E_{2}-v_{1} B\right) \partial_{v_{2}} f=0, \\
\rho=-\int(f-F(v)) d v \\
\nabla \cdot E=\rho, \\
\nabla \times E=0 .
\end{array}\right.
$$

Here $f$ gives the density in phase space of mobile negative ions with mass one and charge minus one. This is assumed to depend on position, $x=\left(x_{1}, x_{2}\right)$, and velocity, $v=\left(v_{1}, v_{2}\right)$. The positive ions have charge positive one, but are taken to have infinite mass so that they form a fixed background density given by $F\left(v_{1}, v_{2}\right)$ (see 6 ). We take $F(v)=0$ for $v_{1} \leq 0 . B=B\left(x_{1}, x_{2}\right)$ is a given magnetic field. The self-consistent magnetic field due to the plasma is neglected. We assume that $E \rightarrow 0$ as $x_{1} \rightarrow-\infty, f-F \rightarrow 0$ as $x_{1} \rightarrow-\infty$ for $v_{1}>0$, and $f-F \rightarrow 0$ as $x_{1} \rightarrow+\infty$ for $v_{1}<0$. We wish to understand the "downwind", i.e., large $x_{1}$, behavior.

In [7 a one space dimensional version of this problem is considered and it is shown that for $B$ small and compactly supported, that $f-F$ and $E$ do not decay to zero as

Received September 28, 2010.

2000 Mathematics Subject Classification. Primary 35L60, 35Q99, 82C21, 82D10.

E-mail address: js5m@andrew.cmu.edu

(C) 2011 Brown University

Reverts to public domain 28 years from publication 
$x_{1} \rightarrow+\infty$. Similar results were shown when a self-consistent magnetic field was included, 8. Thus it is reasonable to expect at most slow decay in higher dimensions.

To make (1.1) more tractable, we will linearize about $B=0$ : Let $g=f-F$. Then we compute that

$$
\left\{\begin{array}{l}
v \cdot \nabla_{x} g-\left(E_{1}+v_{2} B\right) \partial_{v_{1}} F-\left(E_{2}-v_{1} B\right) \partial_{v_{2}} F=0, \\
\rho=-\int g d v \\
\nabla \cdot E=\rho \\
\nabla \times E=0 .
\end{array}\right.
$$

We make the following standing assumptions throughout. Of $F$ we assume that $0 \leq$ $F \in C^{3}\left(\mathbb{R}^{2}\right)$ with $F$ and $|\nabla F|$ in $L^{1}\left(\mathbb{R}^{2}\right)$ and there exist $\varepsilon>0$ and $C>0$ such that

$$
\begin{gathered}
v_{1} \leq \varepsilon \Rightarrow F\left(v_{1}, v_{2}\right)=0 \quad \forall v_{2}, \\
F(v) \leq C|v|^{-1-\varepsilon} \text { and }\left|v_{1} \partial_{v_{2}} F-v_{2} \partial_{v_{1}} F\right| \leq C v_{1}^{-\varepsilon}|v|^{-2}\left|v_{2}\right|, \\
F\left(v_{1},-v_{2}\right)=F\left(v_{1}, v_{2}\right) \quad \forall v, \\
F(v) \neq 0 \text { and } v_{2} \neq 0 \Rightarrow v_{2} \partial_{v_{2}} F(v)<0 .
\end{gathered}
$$

It is also assumed that $B \in C\left(\mathbb{R}^{2}\right), g \in C^{1}\left(\mathbb{R}^{4}\right), \rho \in C^{1}\left(\mathbb{R}^{2}\right), E \in C^{1}\left(\mathbb{R}^{2}\right)$ are all bounded and satisfy (1.2) with

$$
\begin{array}{r}
\left|E\left(x_{1}, x_{2}\right)\right|+\left|\rho\left(x_{1}, x_{2}\right)\right|\left|x_{1}\right| \leq C\left|x_{1}\right|^{-1-\varepsilon} \text { for } x_{1} \leq 0, \\
g(x+s v, v) \rightarrow 0 \text { as } s \rightarrow-\infty \quad \forall x, v, \\
x_{1} \leq 0 \Rightarrow B\left(x_{1}, x_{2}\right)=0 \quad \forall x_{2}, \\
|B(x)| \leq C|x|^{-2-\varepsilon}, \\
v \mapsto g(x, v) \text { is in } L^{1}\left(\mathbb{R}^{2}\right) \forall x, \\
\int\left|\rho\left(x_{1}, x_{2}\right)\right| d x_{2} \leq C e^{r x_{1}}
\end{array}
$$

for some

$$
r>\sup _{w} \int_{0}^{\infty} \tau^{-1} F\left(\tau^{-1} w\right) d \tau
$$

It will be shown that

$$
\rho\left(x_{1}, x_{2}\right)=0 \text { for } x_{1} \leq 0 .
$$

ThEOREM 1.1. In addition to the standing assumptions (1.3) through (1.13) suppose $F$ is of the form

$$
F(v)=A\left(v_{1}\right)\left(1+\left(\frac{v_{2}}{v_{1}}\right)^{2}\right)^{-1} .
$$

Then

$$
\rho(x)=-a_{2} \int_{0}^{x_{1}} \int \frac{\left(x_{2}-\tilde{x}_{2}\right) \cos \left(\left(x_{1}-\tilde{x}_{1}\right) \sqrt{\pi a_{1}}\right)}{\left(x_{1}-\tilde{x}_{1}\right)^{2}+\left(x_{2}-\tilde{x}_{2}\right)^{2}} B(\tilde{x}) d \tilde{x}_{2} d \tilde{x}_{1}
$$


for $x_{1}>0$, where

$$
a_{1}=\int_{0}^{\infty} A\left(\tilde{w}_{1}\right) \tilde{w}_{1}^{-1} d \tilde{w}_{1}
$$

and

$$
a_{2}=\int_{0}^{\infty} A\left(\tilde{w}_{1}\right) d \tilde{w}_{1}
$$

Comment. If $A\left(v_{1}\right)$ is compactly supported, then (1.4) holds.

Corollary 1.1. Under the assumptions of Theorem 1.1,

$$
|\rho(x)| \leq 4 \pi a_{2}\left(\sup \left(x_{1}^{2}|B|\right)+\int|B| d x\right) x_{1}^{-1} .
$$

Moreover, the decay rate of $x_{1}^{-1}$ is optimal.

The next theorem shows that for $F$ compactly supported, the decay rate for $\rho$ is even slower.

TheOREM 1.2. In addition to the standing assumptions (1.3) through (1.13), assume that $F$ is compactly supported. Then there exists $B \in C^{\infty}\left(\mathbb{R}^{2}\right)$ with

$$
\lim _{|x| \rightarrow \infty}|x|^{p}|B(x)|=0
$$

for every $p>0$, such that

$$
\sup _{x_{2}}\left|\rho\left(x_{1}, x_{2}\right)\right| \geq C x_{1}^{-\frac{1}{2}}
$$

for some $C>0$ and all $x_{1}>C$.

Although (1.2) is linearized about $B=0$, it seems likely that slow decay rates apply to the full nonlinear problem as well. In fact, the magnetic tail of the earth has been observed to extend beyond 200 earth radii downwind, $[5$.

Linearization of the dynamic problem about $f=F$ (with $B=0$ ) has been studied extensively. The decay in $\rho$ for large time was first observed by Landau, 3. A more mathematical treatment is contained in [9]. Estimates of decay rates are established in [1] and 2] and (as in Theorems 1.1 and 1.2 above) they depend on $F$. We comment that equation (2.2) of this paper (in $x_{1}, x_{2}$ ) and equation (7) of [1] (in $t, x$ ) have a similar structure. This was unexpected since $E$ is derived from a two-dimensional density here and a one-dimensional density in [1]. The conservation laws used in [1] do not seem to have analogues here. Lemma 2 of [1] and Theorem 1.1 here are closely related.

This paper is organized as follows: The integral equation for $\rho$ is derived in Section 2. In Section 3 we take the Laplace transform in $x_{1}$ and the Fourier transform in $x_{2}$. Section 4 contains the proofs of Theorem 1.1 and its corollary. The proof of Theorem 1.2 in Section 5 uses the stationary phase approximation to get pointwise estimates. We mention that the non-decay results of [1] are in the $L_{2}$ norm and did not require use of the stationary phase approximation.

The following notation is used:

$$
\left\langle a_{1}, a_{2}\right\rangle \wedge\left\langle b_{1}, b_{2}\right\rangle=a_{1} b_{2}-a_{2} b_{1} .
$$


\|\|$_{p}$ is the $L_{p}$ norm. Also, $C$ denotes a positive generic constant which may change from line to line and may depend on $F$ but not on $B, x$, or $v$.

2. A convolution equation for $\rho$. Consider (1.2) with the assumptions (1.3) through (1.13). Then

$$
\begin{aligned}
g(x, v) & =\int_{-\infty}^{0} \frac{d}{d s}(g(x+s v, v)) d s \\
& =\int_{-\infty}^{0} v \cdot \nabla_{x} g(x+s v, v) d s \\
& =\left.\int_{0}^{\infty}\left(\partial_{v_{1}} F\left(E_{1}+v_{2} B\right)+\partial_{v_{2}} F\left(E_{2}-v_{1} B\right)\right)\right|_{x-\tau v} d \tau
\end{aligned}
$$

and

$$
\begin{aligned}
\rho(x) & =-\iint_{0}^{\infty}(\nabla F(v) \cdot E(x-\tau v)-v \wedge \nabla F(v) B(x-\tau v)) d \tau d v \\
& =\int_{0}^{\infty} \int\left[F(v) \nabla_{v} \cdot(E(x-\tau v))+v \wedge \nabla F(v) B(x-\tau v)\right] d v d \tau \\
& =\int_{0}^{\infty} \int[-\tau F(v) \rho(x-\tau v)+v \wedge \nabla F(v) B(x-\tau v)] d v d \tau .
\end{aligned}
$$

Letting

$$
k(w)=\int_{0}^{\infty} \tau^{-1} F\left(\tau^{-1} w\right) d \tau
$$

and

$$
k_{1}(w)=\int_{0}^{\infty}\left(\tau^{-1} w\right) \wedge \nabla F\left(\tau^{-1} w\right) \tau^{-2} d \tau
$$

(2.1) becomes (letting $w=\tau v$ )

$$
\rho(x)=-\int k(w) \rho(x-w) d w+\int k_{1}(w) B(x-w) d w .
$$

Note that $k(w)=k_{1}(w)=0$ if $w_{1} \leq 0$. Since $B=0$ on $(-\infty, 0] \times \mathbb{R}$,

$$
\int k_{1}(w) B(x-w) d w=\int_{0}^{\infty} \int k_{1}(w) B(x-w) d w_{2} d w_{1}=0
$$

for $x_{1} \leq 0$. Hence (2.2) becomes

$$
\rho(x)=-\int_{0}^{\infty} \int k(w) \rho(x-w) d w_{2} d w_{1},
$$

and we claim that $\rho=0$ on $(-\infty, 0] \times \mathbb{R}$. To show this let

$$
\|\rho\|=\sup _{x_{1} \leq 0} e^{-r x_{1}} \int\left|\rho\left(x_{1}, x_{2}\right)\right| d x_{2},
$$


where $r$ is from (1.13). Then for $x_{1} \leq 0$,

$$
\begin{aligned}
|\rho(x)| & \leq\|k\|_{\infty} \int_{0}^{\infty} \int|\rho(x-w)| d w_{2} d w_{1} \\
& \leq\|k\|_{\infty} \int_{0}^{\infty}\|\rho\| e^{r\left(x_{1}-w_{1}\right)} d w_{1} \\
& =\frac{\|k\|_{\infty}}{r}\|\rho\| e^{r x_{1}}
\end{aligned}
$$

and

$$
\|\rho\| \leq \frac{\|k\|_{\infty}}{r}\|\rho\|
$$

Since $r>\|k\|_{\infty},\|\rho\|=0$ follows.

Defining

$$
(a * b)(x)=\int_{0}^{x_{1}} \int a(w) b(x-w) d w_{2} d w_{1},
$$

(2.2) may be written as

$$
\rho=-k * \rho+k_{1} * B
$$

for $x_{1} \geq 0$.

Note that $k(w)=k_{1}(w)=0$ if $w_{1} \leq 0$. For $w_{1}>0$ (and letting $\left.\tilde{w}_{1}=\frac{w_{1}}{\tau}\right)$,

$$
\begin{aligned}
k(w) & =\int_{0}^{\infty} \tau^{-1} F\left(\tau^{-1} w\right) d \tau \\
& =\int_{0}^{\infty} F\left(\tilde{w}_{1}, \frac{w_{2}}{w_{1}} \tilde{w}_{1}\right) \tilde{w}_{1}^{-1} d \tilde{w}_{1}=H\left(\frac{w_{2}}{w_{1}}\right),
\end{aligned}
$$

where

$$
H(\lambda)=\int_{0}^{\infty} F\left(\tilde{w}_{1}, \lambda \tilde{w}_{1}\right) \tilde{w}_{1}^{-1} d \tilde{w}_{1}
$$

Similarly, for $w_{1}>0$,

$$
\begin{aligned}
k_{1}(w) & =\int_{0}^{\infty}\left(\tau^{-1} w\right) \wedge \nabla F\left(\tau^{-1} w\right) \tau^{-2} d \tau \\
& =w_{1}^{-1} H_{1}\left(\frac{w_{2}}{w_{1}}\right)
\end{aligned}
$$

where

$$
H_{1}(\lambda)=\int_{0}^{\infty}\left\langle\tilde{w}_{1}, \lambda \tilde{w}_{1}\right\rangle \wedge \nabla F\left(\tilde{w}_{1}, \lambda \tilde{w}_{1}\right) d \tilde{w}_{1} .
$$

Note that $H$ is even and $H_{1}$ is odd. 
3. Transforms. We will use the Laplace transform in $x_{1}$ :

$$
\mathcal{L} h(s)=\int_{0}^{\infty} h\left(x_{1}\right) e^{-s x_{1}} d x_{1}
$$

and the Fourier transform in $x_{2}$ :

$$
\hat{h}(\xi)=\int h\left(x_{2}\right) e^{-i \xi x_{2}} d x_{2} .
$$

Then for $s=s_{1}+i s_{2}$ with $s_{1}>0$ and $\xi \neq 0$,

$$
\begin{aligned}
\mathcal{L} \hat{k}(s, \xi) & =\int_{0}^{\infty} \int H\left(\frac{x_{2}}{x_{1}}\right) e^{-x_{1} s-i x_{2} \xi} d x_{2} d x_{1} \\
& =\int_{0}^{\infty} \int H(\lambda) e^{-(s+i \xi \lambda) x_{1}} x_{1} d \lambda d x_{1} \\
& =\int H(\lambda) \int_{0}^{\infty} x_{1} e^{-(s+i \xi \lambda) x_{1}} d x_{1} d \lambda \\
& =\int H(\lambda)(s+i \xi \lambda)^{-2} d \lambda=\xi^{-2} \mathcal{H}\left(\frac{s}{\xi}\right),
\end{aligned}
$$

where

$$
\mathcal{H}(s)=\int H(\lambda)(s+i \lambda)^{-2} d \lambda .
$$

Similarly

$$
\begin{aligned}
\mathcal{L} \hat{k}_{1}(s, \xi) & =\int_{0}^{\infty} \int x_{1}^{-1} H_{1}\left(\frac{x_{2}}{x_{1}}\right) e^{-x_{1} s-i \xi x_{2}} d x_{2} d x_{1} \\
& =\int H_{1}(\lambda) \int_{0}^{\infty} x_{1}^{-1} e^{-(s+i \xi \lambda) x_{1}} x_{1} d x_{1} d \lambda \\
& =\int H_{1}(\lambda)(s+i \xi \lambda)^{-1} d \lambda=\xi^{-1} \mathcal{H}_{1}\left(\frac{s}{\xi}\right),
\end{aligned}
$$

where

$$
\mathcal{H}_{1}(s)=\int H_{1}(\lambda)(s+i \lambda)^{-1} d \lambda .
$$

Hence (2.3) yields

$$
\mathcal{L} \hat{\rho}=-\xi^{-2} \mathcal{H}\left(\frac{s}{\bar{\xi}}\right) \mathcal{L} \hat{\rho}+\xi^{-1} \mathcal{H}_{1}\left(\frac{s}{\bar{\xi}}\right) \mathcal{L} \hat{B} .
$$

Let's collect some observations about $\mathcal{H}$ and $\mathcal{H}_{1}$. Both are analytic on $\mathbb{C} \backslash\left\{s_{2} i\right.$ : $\left.s_{2} \in \mathbb{R}\right\}$. Also

$$
\begin{gathered}
\mathcal{H}(-s)=\mathcal{H}(s), \\
\mathcal{H}_{1}(-s)=\mathcal{H}_{1}(s), \\
\overline{\mathcal{H}(s)}=\mathcal{H}(\bar{s}),
\end{gathered}
$$

and

$$
\overline{\mathcal{H}_{1}(s)}=-\mathcal{H}_{1}(\bar{s})
$$


for $\operatorname{im}(s) \neq 0$. It follows that

$$
\begin{aligned}
\mathcal{H}(-\bar{s}) & =\overline{\mathcal{H}(s)}, \\
\mathcal{H}_{1}(-\bar{s}) & =-\overline{\mathcal{H}_{1}(s)}
\end{aligned}
$$

and that for $s_{1} \in \mathbb{R} \backslash\{0\}$,

$$
\begin{aligned}
\overline{\mathcal{H}\left(s_{1}\right)} & =\mathcal{H}\left(s_{1}\right), \\
\overline{\mathcal{H}_{1}\left(s_{1}\right)} & =-\mathcal{H}_{1}\left(s_{1}\right),
\end{aligned}
$$

so $\mathcal{H}\left(s_{1}\right)$ is real and $\mathcal{H}_{1}\left(s_{1}\right)$ is pure imaginary. An elementary calculation shows that

$$
i m\left(\mathcal{H}\left(s_{1}+i s_{2}\right)\right)=4 s_{1} s_{2} \int_{0}^{\infty} \frac{\lambda H^{\prime}(\lambda) d \lambda}{\left(s_{1}^{2}+\left(s_{2}+\lambda\right)^{2}\right)\left(s_{1}^{2}+\left(s_{2}-\lambda\right)^{2}\right)}
$$

so, using (1.6),

$$
i m\left(\mathcal{H}\left(s_{1}+i s_{2}\right)\right) \neq 0 \text { if } s_{1} s_{2} \neq 0 .
$$

Also for $s_{1} \in \mathbb{R} \backslash\{0\}$ (since $H$ is even and (1.6) holds),

$$
\begin{aligned}
\mathcal{H}\left(s_{1}\right) & =\int H(\lambda) \frac{s_{1}^{2}-\lambda^{2}-2 i \lambda s_{1}}{\left(s_{1}^{2}+\lambda^{2}\right)^{2}} d \lambda \\
& =\int H(\lambda) \frac{d}{d \lambda}\left(\frac{\lambda}{s_{1}^{2}+\lambda^{2}}\right) d \lambda=-\int \frac{\lambda H^{\prime}(\lambda)}{s_{1}^{2}+\lambda^{2}}>0 .
\end{aligned}
$$

It follows that

$$
1+\xi^{-2} \mathcal{H}\left(\frac{s}{\xi}\right) \neq 0
$$

for $r e(s) \neq 0$ and we may define

$$
\mathcal{R}(s, \xi)=\frac{\xi^{-1} \mathcal{H}_{1}\left(\frac{s}{\xi}\right)}{1+\xi^{-2} \mathcal{H}\left(\frac{s}{\xi}\right)}
$$

for $r e(s) \neq 0$. Note that $\mathcal{R}$ is analytic in $s$ and by (3.3) that

$$
\mathcal{L} \hat{\rho}=\mathcal{R} \mathcal{L} \hat{B} .
$$

It follows from (3.4) and (3.5) that $\mathcal{R}(s,-\xi)=-\mathcal{R}(s, \xi)$ and hence

$$
\mathcal{R}(s, \xi)=\frac{\xi^{-1} \mathcal{H}_{1}\left(\frac{s}{|\xi|}\right)}{1+\xi^{-2} \mathcal{H}\left(\frac{s}{|\xi|}\right)} .
$$

Similarly, it follows that

$$
\mathcal{R}(-\bar{s}, \xi)=-\overline{\mathcal{R}(s, \xi)}
$$


4. The Proof of Theorem 1.1. Suppose $F$ has the form

$$
F(v)=A\left(v_{1}\right) B\left(\frac{v_{2}}{v_{1}}\right) .
$$

Then from (2.5),

$$
H(\lambda)=\int_{0}^{\infty} A\left(\tilde{w}_{1}\right) B(\lambda) \tilde{w}_{1}^{-1} d \tilde{w}_{1}=a_{1} B(\lambda),
$$

where

$$
a_{1}=\int_{0}^{\infty} A\left(\tilde{w}_{1}\right) \tilde{w}_{1}^{-1} d \tilde{w}_{1} .
$$

Similarly, from (2.6) an elementary calculation reveals that

$$
H_{1}(\lambda)=a_{2}\left(\left(1+\lambda^{2}\right) B^{\prime}(\lambda)+\lambda B(\lambda)\right),
$$

where

$$
a_{2}=\int_{0}^{\infty} A\left(\tilde{w}_{1}\right) d \tilde{w}_{1} .
$$

Taking $B(\lambda)=\left(1+\lambda^{2}\right)^{-1}$ leads to

$$
H_{1}(\lambda)=-a_{2} \lambda\left(1+\lambda^{2}\right)^{-1} .
$$

Next (3.1) and (3.2) become

$$
\mathcal{H}(s)=a_{1} \int\left(1+\lambda^{2}\right)^{-1}(s+i \lambda)^{-2} d \lambda
$$

and

$$
\mathcal{H}_{1}(s)=-a_{2} \int \lambda\left(1+\lambda^{2}\right)^{-1}(s+i \lambda)^{-1} d \lambda .
$$

These may be evaluated using residues. For $r e(s)>0, \lambda \mapsto\left(1+\lambda^{2}\right)^{-1}(s+i \lambda)^{-2}$ is analytic with poles at $\pm i$ and $i s$. is is in the upper half-plane and so

$$
\begin{aligned}
\mathcal{H}(s) & =-\oint_{|\lambda+i|=\delta} \frac{a_{1} d \lambda}{\left(1+\lambda^{2}\right)(s+i \lambda)^{2}} \\
& =-\left.2 \pi i \frac{a_{1}}{(\lambda-i)(s+i \lambda)^{2}}\right|_{\lambda=-i}=\frac{\pi a_{1}}{(s+1)^{2}}
\end{aligned}
$$

for $\delta \in(0,1)$. Similarly

$$
\begin{aligned}
\mathcal{H}_{1}(s) & =-\oint_{|\lambda+i|=\delta} \frac{\left(-a_{2}\right) \lambda d \lambda}{\left(1+\lambda^{2}\right)(s+i \lambda)} \\
& =\left.2 \pi i \frac{a_{2} \lambda}{(\lambda-i)(s+i \lambda)}\right|_{\lambda=-i}=\frac{\pi a_{2} i}{s+1} .
\end{aligned}
$$

Substitution into (3.7) yields

$$
\mathcal{R}(s, \xi)=\frac{\pi a_{2} i(s+|\xi|)}{(s+|\xi|)^{2}+\pi a_{1}} \operatorname{sign}(\xi)
$$

for $r e(s)>0$ and $\xi \neq 0$. Here $\operatorname{sign}(\xi)=\frac{\xi}{|\xi|}$. 
The inverse Laplace transform of $\mathcal{R}$ may be computed by another residue calculation. Let $\mathcal{C}\left(s_{1}\right)=\left\{s_{1}+i s_{2}: s_{2} \in \mathbb{R}\right\}$. Then for any $s_{1}>0$ and $x_{1}>0$,

$$
\begin{aligned}
& \mathcal{L}^{-1} \mathcal{R}\left(x_{1}, \xi\right)=\frac{1}{2 \pi i} \int_{\mathcal{C}\left(s_{1}\right)} \mathcal{R}(s, \xi) e^{s x_{1}} d s \\
& =\frac{\pi a_{2} i \operatorname{sign}(\xi)}{2 \pi i} \int_{\mathcal{C}\left(s_{1}\right)} \frac{(s+|\xi|) e^{s x_{1}} d s}{(s+|\xi|)^{2}+\pi a_{1}} \\
& =\frac{\pi a_{2} i \operatorname{sign}(\xi)}{2 \pi i}\left(\oint_{|s+| \xi\left|-i \sqrt{\pi a_{1}}\right|=\delta} \frac{(s+|\xi|) e^{s x_{1}} d s}{(s+|\xi|)^{2}+\pi a_{1}}+\oint_{|s+| \xi\left|+i \sqrt{\pi a_{1}}\right|=\delta} \frac{(s+|\xi|) e^{s x_{1}} d s}{(s+|\xi|)^{2}+\pi a_{1}}\right) \\
& =\pi a_{2} i \operatorname{sign}(\xi)\left(\left.\frac{(s+|\xi|) e^{s x_{1}}}{s+|\xi|+i \sqrt{\pi a_{1}}}\right|_{s=-|\xi|+i \sqrt{\pi a_{1}}}+\left.\frac{(s+|\xi|) e^{s x_{1}}}{s+|\xi|-i \sqrt{\pi a_{1}}}\right|_{s=-|\xi|-i \sqrt{\pi a_{1}}}\right) \\
& =\pi a_{2} i \operatorname{sign}(\xi) e^{-x_{1}|\xi|} \cos \left(x_{1} \sqrt{\pi a_{1}}\right) .
\end{aligned}
$$

Denoting the inverse Fourier transform by $\mathcal{F}^{-1}$ we have

$$
\mathcal{F}^{-1} \mathcal{L}^{-1} \mathcal{R}(x)=\frac{1}{2 \pi} \int \mathcal{L}^{-1} \mathcal{R}\left(x_{1}, \xi\right) e^{i \xi x_{2}} d \xi .
$$

Substitution of (4.1) and a direct calculation leads to

$$
\mathcal{F}^{-1} \mathcal{L}^{-1} \mathcal{R}(x)=-\frac{a_{2} x_{2} \cos \left(x_{1} \sqrt{\pi a_{1}}\right)}{x_{1}^{2}+x_{2}^{2}}
$$

From (3.6) it follows that

$$
\rho=\left(\mathcal{F}^{-1} \mathcal{L}^{-1} \mathcal{R}\right) * B
$$

so Theorem 1.1 follows.

To prove the corollary note that

$$
\begin{aligned}
|\rho(x)| & \leq \int_{0}^{x_{1}} \int \frac{a_{2}\left|x_{2}-\tilde{x}_{2}\right|}{\left(x_{1}-\tilde{x}_{1}\right)^{2}+\left(x_{2}-\tilde{x}_{2}\right)^{2}}|B(\tilde{x})| d \tilde{x}_{2} d \tilde{x}_{1} \\
& \leq a_{2} \int_{0}^{x_{1}} \int \frac{|B(\tilde{x})|}{r} d \tilde{x}_{2} d \tilde{x}_{1},
\end{aligned}
$$

where

$$
r=\sqrt{\left(x_{1}-\tilde{x}_{1}\right)^{2}+\left(x_{2}-\tilde{x}_{2}\right)^{2}} .
$$


Note that $r<\frac{1}{2} x_{1} \Rightarrow x_{1}-\tilde{x}_{1}<\frac{1}{2} x_{1} \Rightarrow \frac{1}{2} x_{1}<\tilde{x}_{1}$, so letting $C_{B}=\sup \left(x_{1}^{2}\left|B\left(x_{1}, x_{2}\right)\right|\right)$ we have

$$
\begin{aligned}
\iint_{r<\frac{1}{2} x_{1}} \frac{|B(\tilde{x})|}{r} d \tilde{x}_{2} d \tilde{x}_{1} & \leq \iint_{r<\frac{1}{2} x_{1}} \frac{C_{B}}{\tilde{x}_{1}^{2}} \frac{d \tilde{x}_{2} d \tilde{x}_{1}}{r} \\
& \leq \frac{C_{B}}{\left(\frac{1}{2} x_{1}\right)^{2}} \iint_{r<\frac{1}{2} x_{1}} \frac{d \tilde{x}_{2} d \tilde{x}_{1}}{r}=\frac{4 C_{B}}{x_{1}^{2}} 2 \pi \frac{1}{2} x_{1}=\frac{4 \pi C_{B}}{x_{1}} .
\end{aligned}
$$

Hence

$$
\begin{aligned}
|\rho(x)| & \leq\left(\frac{4 \pi C_{B}}{x_{1}}+\int_{0}^{x_{1}} \int \frac{|B(\tilde{x})|}{\frac{1}{2} x_{1}} d \tilde{x}_{2} d \tilde{x}_{1}\right) a_{2} \\
& \leq \frac{\left(4 \pi C_{B}+2\|B\|_{L^{1}}\right) a_{2}}{x_{1}} .
\end{aligned}
$$

To show that this rate is sharp, let

$$
\omega=\sqrt{\pi a_{1}}
$$

and assume that $B$ is positive on the set $\left\{\left(x_{1}, x_{2}\right): 0<x_{1}<\omega^{-1}\right.$ and $\left.\left|x_{2}\right|<\omega^{-1}\right\}$ and zero everywhere else. Consider $x=\left(2 n \pi \omega^{-1}, 2 n \pi \omega^{-1}\right)$, where $n$ is a positive integer. Then

$$
\begin{aligned}
-\frac{\rho(x)}{a_{2}} & =\int_{0}^{\omega^{-1}} \int_{-\omega^{-1}}^{\omega^{-1}} \frac{\left(x_{2}-\tilde{x}_{2}\right) \cos \left(\omega\left(x_{1}-\tilde{x}_{1}\right)\right)}{\left(x_{1}-\tilde{x}_{1}\right)^{2}+\left(x_{2}-\tilde{x}_{2}\right)^{2}} B(\tilde{x}) d \tilde{x}_{2} d \tilde{x}_{1} \\
& \geq \int_{0}^{\omega^{-1}} \int_{-\omega^{-1}}^{\omega^{-1}} \frac{\left(2 n \pi \omega^{-1}-\omega^{-1}\right) \cos (1) B(\tilde{x})}{\left(2 n \pi \omega^{-1}\right)^{2}+\left(2 n \pi \omega^{-1}+\omega^{-1}\right)^{2}} d \tilde{x}_{2} d \tilde{x}_{1} \\
& \geq \frac{C\|B\|_{L^{1}}}{x_{1}}
\end{aligned}
$$

5. The Proof of Theorem 1.2. In this section we assume that $F$ has compact support and hence there exists $r_{H}>0$ such that $|\lambda| \geq r_{H} \Rightarrow H(\lambda)=H_{1}(\lambda)=0$. Then $\mathcal{H}$ and $\mathcal{H}_{1}$ are analytic on $\mathcal{C} \backslash\left\{i s_{2}:\left|s_{2}\right| \leq r_{H}\right\}$. It is necessary to compute the limits of $\mathcal{H}$ and $\mathcal{H}_{1}$ as $r e(s) \rightarrow 0^{+}$(and $\left.|i m(s)| \leq r_{H}\right)$.

The following notation will be used:

$$
\sigma_{*}\left(s_{2}\right)=\frac{1}{\pi} \lim _{\delta \rightarrow 0^{+}}\left(\int_{-\infty}^{-s_{2}-\delta} \frac{\sigma(\lambda)}{s_{2}+\lambda} d \lambda+\int_{-s_{2}+\delta}^{\infty} \frac{\sigma(\lambda)}{s_{2}+\lambda} d \lambda\right)
$$

whenever this limit exists. Also consider $\sigma \in C^{1}(\mathbb{R})$ with $|\sigma(\lambda)|+\left|\lambda \sigma^{\prime}(\lambda)\right| \leq C|\lambda|^{-\delta}$ for some $C, \delta>0$. For $s=s_{1}+i s_{2}$ with $s_{1}>0$, define $\arg (s) \in\left(-\frac{\pi}{2}, \frac{\pi}{2}\right)$ by $s=|s| e^{i \arg (s)}$. 
Then

$$
\begin{aligned}
\int \sigma(\lambda)\left(s_{2}+i \lambda\right)^{-1} d \lambda & =\int \sigma(\lambda)(-i) \frac{d}{d \lambda}[\log (s+i \lambda)] d \lambda \\
& =i \int \sigma^{\prime}(\lambda)(\ln |s+\lambda|+i \arg (s+i \lambda)) d \lambda \\
& \underset{s_{1} \rightarrow 0^{+}}{\longrightarrow} i \int \sigma^{\prime}(\lambda)\left(\ln \left|s_{2}+\lambda\right|+i \frac{\pi}{2} \operatorname{sign}\left(s_{2}+\lambda\right)\right) d \lambda \\
& =\pi\left(\sigma\left(-s_{2}\right)-i \sigma_{*}\left(s_{2}\right)\right) .
\end{aligned}
$$

(5.1) will be applied to $\mathcal{H}$ and to $\mathcal{H}_{1}$. Recall that $H$ is even and hence $H^{\prime}$ is odd. By (3.1) and (5.1),

$$
\begin{aligned}
& \mathcal{H}(s)= \int H(\lambda) \frac{d}{d \lambda}\left[i(s+i \lambda)^{-1}\right] d \lambda \\
&=-i \int H^{\prime}(\lambda)(s+i \lambda)^{-1} d \lambda \\
& \underset{s_{1} \rightarrow 0^{+}}{\longrightarrow}-i \pi\left(H^{\prime}\left(-s_{2}\right)-i\left(H^{\prime}\right)_{*}\left(s_{2}\right)\right) \\
&=\pi\left(-\left(H^{\prime}\right)_{*}\left(s_{2}\right)+i H^{\prime}\left(s_{2}\right)\right) .
\end{aligned}
$$

Similarly, by (3.2) and (5.1),

$$
\mathcal{H}_{1}(s) \underset{s_{1} \rightarrow 0^{+}}{\longrightarrow} \pi\left(H_{1}\left(-s_{2}\right)-i H_{1 *}\left(s_{2}\right)\right)=-\pi\left(H_{1}\left(s_{2}\right)+i H_{1 *}\left(s_{2}\right)\right) .
$$

Let

$$
\mathcal{R}_{+}\left(s_{2}, \xi\right)=\frac{-\pi \xi\left(H_{1}\left(\frac{s_{2}}{|\xi|}\right)+i H_{1 *}\left(\frac{s_{2}}{|\xi|}\right)\right)}{\xi^{2}-\pi\left(H^{\prime}\right)_{*}\left(\frac{s_{2}}{|\xi|}\right)+i \pi H^{\prime}\left(\frac{s_{2}}{|\xi|}\right)} .
$$

Then by (3.7), (5.2), and (5.3),

$$
\mathcal{R}(s, \xi) \rightarrow \mathcal{R}_{+}\left(s_{2}, \xi\right) \text { as } s_{1} \rightarrow 0^{+} .
$$

Consider $s=s_{2} i$ with $\left|\frac{s_{2}}{\xi}\right|>r_{H}$, recalling that $\mathcal{H}$ and $\mathcal{H}_{1}$ are analytic at these points. Define

$$
h(z)=\int H(\lambda)(\lambda+z)^{-2} d \lambda
$$

for $|z|>r_{H}$. Then $h$ is even, decreasing on $\left(r_{H}, \infty\right)$, and

$$
\pi\left(H^{\prime}\right)_{*}\left(\frac{s_{2}}{|\xi|}\right)=\int H(\lambda)\left(\frac{s_{2}}{|\xi|}+\lambda\right)^{2} d \lambda=h\left(\frac{s_{2}}{|\xi|}\right) .
$$

Note that

$$
\mathcal{R}(s, \xi)=\mathcal{R}_{+}\left(s_{2}, \xi\right)=\frac{-\pi \xi i H_{1 *}\left(\frac{s_{2}}{|\xi|}\right)}{\xi^{2}-h\left(\frac{s_{2}}{|\xi|}\right)}
$$


for $s=s_{2} i$ with $\left|\frac{s_{2}}{\xi}\right|>r_{H}$. For $|\xi|>\sqrt{h\left(r_{H}\right)}$ we have $\xi^{2}-h\left(\frac{s_{2}}{|\xi|}\right)>0$ on $\left|\frac{s_{2}}{\xi}\right|>r_{H}$; hence $\mathcal{R}$ is analytic (in $s$ ) on $\mathbb{C} \backslash\left\{i s_{2}:\left|\frac{s_{2}}{\xi}\right| \leq r_{H}\right\}$. For $|\xi|<\sqrt{h\left(r_{H}\right)}, \mathcal{R}$ has poles at $s= \pm p(\xi) i$, where

$$
p(\xi)=|\xi| h^{-1}\left(\xi^{2}\right)
$$

and $h^{-1}$ is the inverse of $h$ restricted to $\left(r_{H}, \infty\right)$.

Let $\mathcal{C}\left(s_{1}\right)=\left\{s_{1}+i s_{2}: s_{2} \in \mathbb{R}\right\}, I_{a<b}=1$ if $a<b$ and 0 otherwise and $\mathcal{R}_{-}\left(s_{2}, \xi\right)=$ $\lim _{s_{1} \rightarrow 0^{-}} \mathcal{R}(s, \xi)$. Since for $x_{1}>0$,

$$
\frac{1}{2 \pi i} \int_{\mathcal{C}\left(s_{1}\right)} \mathcal{R}(s, \xi) e^{s x_{1}} d s= \begin{cases}0 & \text { if } s_{1}<0 \\ \mathcal{L}^{-1} \mathcal{R}\left(x_{1}, \xi\right) & \text { if } s_{1}>0\end{cases}
$$

we have for $\delta>0$ sufficiently small,

$$
\begin{aligned}
\mathcal{L}^{-1} \mathcal{R}\left(x_{1}, \xi\right)= & \frac{1}{2 \pi i} \int_{-r_{H}|\xi|}^{r_{H}|\xi|}\left(\mathcal{R}_{+}-\mathcal{R}_{-}\right) e^{i s_{2} x_{1}} i d s_{2} \\
& +\frac{1}{2 \pi i} I_{|\xi|<\sqrt{h\left(r_{H}\right)}}\left(\oint_{|s-p(\xi) i|=\delta} \mathcal{R} e^{s x_{1}} d s+\oint_{|s+p(\xi) i|=\delta} \mathcal{R} e^{s x_{1}} d s\right) .
\end{aligned}
$$

By (3.8),

$$
\mathcal{R}_{+}-\mathcal{R}_{-}=\mathcal{R}_{+}+\overline{\mathcal{R}}_{+}=2 \operatorname{re}\left(\mathcal{R}_{+}\right) .
$$

Let

$$
\mathcal{R}_{0}(z, \xi)=\frac{-\xi\left(H_{1}(z)\left(\xi^{2}-\pi\left(H^{\prime}\right)_{*}(z)\right)+\pi H_{1 *}(z) H^{\prime}(z)\right)}{\left(\xi^{2}-\pi\left(H^{\prime}\right)_{*}(z)\right)^{2}+\left(\pi H^{\prime}(z)\right)^{2}} .
$$

Then

$$
\begin{aligned}
& \frac{1}{2 \pi} \int_{-r_{H}|\xi|}^{r_{H}|\xi|}\left(\mathcal{R}_{+}-\mathcal{R}_{-}\right) e^{i s_{2} x_{1}} d s_{2} \\
& =\int_{-r_{H}|\xi|}^{r_{H}|\xi|} \mathcal{R}_{0}\left(\frac{s_{2}}{|\xi|}, \xi\right) e^{i s_{2} x_{1}} d s_{2} \\
& =\int_{-r_{H}}^{r_{H}} \mathcal{R}_{0}(z, \xi) e^{i|\xi| z x_{1}}|\xi| d z .
\end{aligned}
$$


The second term of (5.5) may be computed using residues. For $|\xi|<\sqrt{h\left(r_{H}\right)}$, (3.7) yields

$$
\begin{aligned}
\frac{1}{2 \pi i} \oint_{|s-p i|=\delta} \mathcal{R} e^{s x_{1}} d s & =\frac{1}{2 \pi i} \oint_{|s-p i|=\delta} \frac{\xi \mathcal{H}_{1}\left(\frac{s}{|\xi|}\right) e^{s x_{1}} d s}{\xi^{2}+\mathcal{H}\left(\frac{s}{|\xi|}\right)} \\
& =\frac{\xi \mathcal{H}_{1}\left(\frac{i p}{|\xi|}\right) e^{i p x_{1}}}{\mathcal{H}^{\prime}\left(\frac{i p}{|\xi|}\right)|\xi|^{-1}} .
\end{aligned}
$$

Since $\frac{p}{|\xi|}>r_{H}$ it follows from (3.1), (3.2), and (5.4) that

$$
\mathcal{H}^{\prime}\left(\frac{i p}{|\xi|}\right)=i h^{\prime}\left(\frac{p}{|\xi|}\right)
$$

and

$$
\mathcal{H}_{1}\left(\frac{i p}{|\xi|}\right)=-i \pi H_{1 *}\left(\frac{p}{|\xi|}\right)
$$

so

$$
\frac{1}{2 \pi i} \oint_{|s-p i|=\delta} \mathcal{R} e^{s x_{1}} d s=-\pi \xi|\xi| \frac{H_{1 *}\left(\frac{p}{|\xi|}\right)}{h^{\prime}\left(\frac{p}{|\xi|}\right)} e^{i p x_{1}} .
$$

In similar fashion, it may be shown that (also using (3.4) and (3.5))

$$
\frac{1}{2 \pi i} \oint_{|s+p i|=\delta} \mathcal{R} e^{s x_{1}} d s=\pi \xi|\xi| \frac{H_{1 *}\left(\frac{p}{|\xi|}\right)}{h^{\prime}\left(\frac{p}{|\xi|}\right)} e^{-i p x_{1}} .
$$

Collecting (5.6), (5.7), and (5.8), (5.5) becomes

$$
\begin{aligned}
\mathcal{L}^{-1} \mathcal{R}\left(x_{1}, \xi\right)= & \int_{-r_{H}}^{r_{H}} \mathcal{R}_{0}(z, \xi) e^{i|\xi| x_{1} z}|\xi| d z \\
& -\pi \xi|\xi| I_{|\xi|<\sqrt{h\left(r_{H}\right)}} \frac{H_{1 *}\left(\frac{p}{|\xi|}\right)}{h^{\prime}\left(\frac{p}{|\xi|}\right)}\left(e^{i p x_{1}}-e^{-i p x_{1}}\right) .
\end{aligned}
$$

Since $\rho=\mathcal{F}^{-1} \mathcal{L}^{-1} \mathcal{R} * B,(5.9)$ allows us to represent the solution. We claim that the first term of (5.9) satisfies

$$
\left|\int_{-r_{H}}^{r_{H}} \mathcal{R}_{0}(z, \xi) e^{i|\xi| x_{1} z}\right| \xi|d z| \leq \frac{C}{x_{1}}
$$

for $|\xi| \leq \frac{1}{2} \sqrt{h\left(r_{H}\right)}$. This estimate seems to break down as $|\xi|$ approaches $\sqrt{h\left(r_{H}\right)}$. Hence the strategy for proving Theorem 1.2 is to choose $B$ with $|\xi|>\frac{1}{2} \sqrt{h\left(r_{H}\right)} \Rightarrow \hat{B}\left(x_{1}, \xi\right)=0$. Then the second term of (5.9) will be analyzed using the stationary phase approximation. 
First we establish (5.10). Note that $\left(H^{\prime}\right)_{*}$ is continuous with $\left(H^{\prime}\right)_{*}(0)<0$ and $\pi\left(H^{\prime}\right)_{*}(z)=h(z)$ for $|z|>r_{H}$. Hence we may choose $r_{1}$ and $r_{2}$ with $0<r_{1}<r_{2}<r_{H}$ such that

$$
\pi\left(H^{\prime}\right)_{*}(z) \leq-C \text { if }|z| \leq r_{1}
$$

and

Let

$$
\pi\left(H^{\prime}\right)_{*}(z) \geq \frac{1}{2} h\left(r_{H}\right) \quad \text { if } r_{2} \leq r \leq r_{H} .
$$

For $|z| \leq r_{1}$

$$
D(z, \xi)=\left(\xi^{2}-\pi\left(H^{\prime}\right)_{*}\right)^{2}+\left(\pi H^{\prime}\right)^{2}
$$

$D \geq\left(\xi^{2}+C\right)^{2} \geq C$.

On $r_{1} \leq|z| \leq r_{2}, H^{\prime}(z) \neq 0$, so by continuity,

$$
D \geq\left(\pi H^{\prime}(z)\right)^{2} \geq C .
$$

For $r_{2} \leq|z| \leq r_{H}$ and $|\xi| \leq \frac{1}{2} \sqrt{h\left(r_{H}\right)}$,

$$
\begin{aligned}
D & \geq\left(\xi^{2}-\pi\left(H^{\prime}\right)_{*}(z)\right)^{2}=\left(\pi\left(H^{\prime}\right)_{*}(z)-\xi^{2}\right)^{2} \\
& \geq\left(\frac{1}{2} h\left(r_{H}\right)-\left(\frac{1}{2} \sqrt{h\left(r_{H}\right)}\right)^{2}\right)^{2}=\frac{1}{16} h^{2}\left(r_{H}\right) .
\end{aligned}
$$

So for $|\xi| \leq \frac{1}{2} \sqrt{h\left(r_{H}\right)}$ we have $D \geq C$ and it follows that

$$
\left|\partial_{z} \mathcal{R}_{0}(z, \xi)\right| \leq C .
$$

Since $\mathcal{R}_{0}\left(\xi, \pm r_{H}\right)=0$ we have

$$
\begin{aligned}
& \left|\int_{-r_{H}}^{r_{H}} \mathcal{R}_{0}(z, \xi) e^{i|\xi| x_{1} z}\right| \xi|d z| \\
& \quad=\left|-\int_{r_{H}}^{r_{H}} \partial_{z} \mathcal{R}_{0}(z, \xi) \frac{e^{i|\xi| x_{1} z}}{i x_{1}} d z\right|
\end{aligned}
$$

and (5.10) follows as claimed.

In preparation for using stationary phase on the second term of (5.9) we claim that

$$
0<p^{\prime}(\xi) \text { and } 0<p^{\prime \prime}(\xi)
$$

for $0<\xi<\sqrt{h\left(r_{H}\right)}$. Define

$$
j(z)=z^{2} h(z)=\int H(\lambda)\left(1+\frac{\lambda}{z}\right)^{-2} d \lambda
$$

and

$$
q(\xi)=\frac{p(\xi)}{\xi} .
$$

Then

$$
\xi^{2}=h(q)
$$


and

$$
p^{2}=\xi^{2} q^{2}=q^{2} h(q)=j(q)
$$

Hence

$$
2 \xi=h^{\prime}(q) q^{\prime}
$$

and

$$
2 p p^{\prime}=j^{\prime}(q) q^{\prime}
$$

But $h^{\prime}(z)<0$ and

$$
\begin{aligned}
j^{\prime}(z) & =2 z^{-2} \int \lambda H(\lambda)\left(1+\frac{\lambda}{z}\right)^{-3} d \lambda \\
& =2 z^{-2} \int_{0}^{r_{H}} \lambda H(\lambda)\left[\left(1+\frac{\lambda}{z}\right)^{-3}-\left(1-\frac{\lambda}{z}\right)^{-3}\right] d \lambda<0
\end{aligned}
$$

so

$$
q^{\prime}=\frac{2 \xi}{h^{\prime}(q)}<0
$$

and

$$
p^{\prime}=\frac{j^{\prime}(q) q^{\prime}}{2 p}>0
$$

Furthermore

$$
p^{\prime}=\frac{j^{\prime}(q)}{2 p} \frac{2 \xi}{h^{\prime}(q)}=\ell(q)
$$

where we define $\ell$ by

$$
\ell(z)=\frac{j^{\prime}(z)}{z h^{\prime}(z)} .
$$

If we can show that $\ell^{\prime}<0$, then $p^{\prime \prime}=\ell^{\prime}(q) q^{\prime}>0$ will follow.

Note that

$$
\ell^{\prime}=\left(\frac{2 z h+z^{2} h^{\prime}}{z h^{\prime}}\right)^{\prime}=\frac{3\left(h^{\prime}\right)^{2}-2 h h^{\prime \prime}}{\left(h^{\prime}\right)^{2}} .
$$

By the Cauchy-Schwarz inequality (and since $H^{\frac{1}{2}}(\lambda)(s+\lambda)^{-1}$ and $H^{\frac{1}{2}}(\lambda)(s+\lambda)^{-2}$ are linearly independent),

$$
\begin{aligned}
3\left(h^{\prime}\right)^{2} & =3\left(\int H(\lambda)(-2)(z+\lambda)^{-3} d \lambda\right)^{2} \\
& =12\left(\int H^{\frac{1}{2}}(\lambda)(z+\lambda)^{-1} H^{\frac{1}{2}}(\lambda)(z+\lambda)^{-2} d \lambda\right)^{2} \\
& <12\left(\int H(\lambda)(z+\lambda)^{-2} d \lambda\right)\left(\int H(\lambda)(z+\lambda)^{-4} d \lambda\right) \\
& =12 h\left(\frac{1}{6} h^{\prime \prime}\right)=2 h h^{\prime \prime} .
\end{aligned}
$$

From (5.12), $\ell^{\prime}<0$ now follows and hence (5.11) follows. 
Let

$$
\xi_{L}=\frac{1}{4} \sqrt{h\left(r_{H}\right)}, \xi_{R}=\frac{1}{2} \sqrt{h\left(r_{H}\right)} .
$$

We wish to choose $\xi_{0} \in\left(\xi_{L}, \xi_{R}\right)$ such that

$$
H_{1 *}\left(\frac{p\left(\xi_{0}\right)}{\xi_{0}}\right) \neq 0
$$

Suppose this were not possible. Then $H_{1 *}$ vanishes on an interval outside of $\left[-r_{H}, r_{H}\right]$. But $H_{1 *}$ is analytic on $\mathbb{C} \backslash\left[-r_{H}, r_{H}\right]$, so $H_{1 *}$ is identically zero. This implies that $H_{1}$ and hence $\mathcal{R}$ and hence $\rho$ vanish for all $B$. This is false, so (5.13) must hold.

Now we may choose $B$. Let $B_{1} \in C_{0}^{\infty}(\mathbb{R})$ with $\varepsilon>0$ and $r_{B}>0$ such that $0<\varepsilon<r_{B}$, $x_{1} \notin\left(\varepsilon, r_{B}\right) \Rightarrow B_{1}\left(x_{1}\right)=0$, and

$$
\hat{B}_{1}\left(p\left(\xi_{0}\right)\right) \neq 0 .
$$

Choose $\beta \in C_{0}^{\infty}(\mathbb{R})$ with $\xi \notin\left(\xi_{L}, \xi_{R}\right) \Rightarrow \beta(\xi)=0$ and

$$
\beta\left(\xi_{0}\right) \neq 0 .
$$

Take

$$
B_{2}\left(x_{2}\right)=\frac{1}{2 \pi} \int \beta(\xi) e^{i \xi x_{2}} d \xi=\mathcal{F}^{-1} \beta\left(x_{2}\right)
$$

and

$$
B(x)=B_{1}\left(x_{1}\right) B_{2}\left(x_{2}\right) .
$$

For $x_{1}>r_{B}$,

$$
\begin{aligned}
\hat{\rho}\left(x_{1}, \xi\right) & =\int_{0}^{x_{1}} \hat{B}\left(\tilde{x}_{1}, \xi\right) \mathcal{L}^{-1} \mathcal{R}\left(x_{1}-\tilde{x}_{1}, \xi\right) d \tilde{x}_{1} \\
& =\int_{0}^{r_{B}} B_{1}\left(\tilde{x}_{1}\right) \beta(\xi) \mathcal{L}^{-1} \mathcal{R}\left(x_{1}-\tilde{x}_{1}, \xi\right) d \tilde{x}_{1} .
\end{aligned}
$$

Let

$$
\mathcal{B}(\xi)=-\pi \xi|\xi| \beta(\xi) \frac{H_{1 *}\left(\frac{p}{|\xi|}\right)}{h^{\prime}\left(\frac{p}{|\xi|}\right)}
$$

and note that by (5.13) and (5.14),

$$
\mathcal{B}\left(\xi_{0}\right) \neq 0
$$


For $x_{1}>2 r_{B}$ and $|\xi|<\frac{1}{2} \sqrt{h\left(r_{H}\right)}$, (15.9) and (5.10) yield

$$
\begin{aligned}
\hat{\rho}\left(x_{1}, \xi\right)= & \int_{0}^{r_{B}} B_{1}\left(\tilde{x}_{1}\right) \beta(\xi)\left[O\left(x_{1}-\tilde{x}_{1}\right)^{-1}\right. \\
\left.-\pi \xi|\xi| \frac{H_{1 *}\left(\frac{p}{|\xi|}\right)}{h^{\prime}\left(\frac{p}{|\xi|}\right)}\left(e^{i p\left(x_{1}-\tilde{x}_{1}\right)}-e^{-i p\left(x_{1}-\tilde{x}_{1}\right)}\right)\right] d \tilde{x}_{1} & \\
= & O\left(x_{1}^{-1}\right)+\mathcal{B}(\xi) \int_{0}^{r_{B}} B_{1}\left(\tilde{x}_{1}\right)\left(e^{i p\left(x_{1}-\tilde{x}_{1}\right)}-e^{-i p\left(x_{1}-\tilde{x}_{1}\right)}\right) d \tilde{x}_{1} \\
& =O\left(x_{1}^{-1}\right)+\mathcal{B}(\xi)\left(\hat{B}_{1}(p) e^{i p x_{1}}-\hat{B}_{1}(-p) e^{-i p x}\right) .
\end{aligned}
$$

Let

$$
\lambda=p^{\prime}\left(\xi_{0}\right)
$$

and consider

$$
2 \pi \rho\left(x_{1},-\lambda x_{1}\right)=O\left(x_{1}^{-1}\right)+\int_{\xi_{L}}^{\xi_{R}} \mathcal{B}(\xi)\left(\hat{B}_{1}(p) e^{i p x_{1}}-\hat{B}_{1}(-p) e^{-i p x_{1}}\right) e^{-i \xi \lambda x_{1}} d \xi .
$$

By (5.11), $(p-\lambda \xi)^{\prime \prime}=p^{\prime \prime}>0$ and by (5.15),

$$
(p-\lambda \xi)^{\prime}=0 \text { if } \xi=\xi_{0} \text {. }
$$

Also by (5.11) and (5.15),

$$
(-p-\lambda \xi)^{\prime}<0
$$

for $\xi \in\left(\xi_{L}, \xi_{R}\right)$. Now by the stationary phase approximation (see, for example, pages 100 and 101 of [4]) we have

$$
2 \pi \rho\left(x_{1},-\lambda x_{1}\right)=O\left(x_{1}^{-1}\right)+C_{1} x_{1}^{\frac{-1}{2}}+O\left(x_{1}^{-1}\right),
$$

where

$$
C_{1}=\mathcal{B}\left(\xi_{0}\right) \hat{B}_{1}\left(p\left(\xi_{0}\right)\right) \sqrt{\frac{2 \pi}{p^{\prime \prime}\left(\xi_{0}\right)}} e^{i\left(\frac{\pi}{4}+x_{1}\left[p\left(\xi_{0}\right)-\xi_{0} \lambda\right]\right)} \neq 0 .
$$

This proves Theorem 2.1 .

\section{REFERENCES}

[1] Glassey, R. and Schaeffer, J., Time Decay for Solutions to the Linearized Vlasov Equation, Trans. Th. Stat. Phys., 23, 411-453 (1994). MR.1264846 (95a:82109)

[2] Glassey, R. and Schaeffer, J., On Time Decay Rates in Landau Damping, Commun. PDE, 20, 647-674 (1995). MR1318084 (95m:35194)

[3] Landau, L. D., On the Vibrations of the Electronic Plasma, Ah. Eksper. Teoret. Fiz, 16, 574-586 (1946). MR0023764 (9:401h)

[4] Olver, F. W. J., "Asymptotics and Special Functions", Academic Press, 1974. MR0435697(55:8655)

[5] Parks, G., "Physics of Space Plasmas", Addison-Wesley, 1991.

[6] Rein, G., A Two-species Plasma in the Limit of Large Ion Mass, Math. Meth. Appl. Sci., 13, 159-167 (1990). MR1066383 (91d:76089)

[7] Schaeffer, J., Steady States for a One Dimensional Model of the Solar Wind, Quart. of Appl. Math., 59, 507-528 (2001). MR1848532(2002j:82114) 
[8] Schaeffer, J., Steady States of the Vlasov-Maxwell System, Quart. of Appl. Math., 63, 619-643 (2005). MR2187923 (2006k:82148)

[9] VanKampen, N. G. and Felderhof, B. U., "Theoretical Methods in Plasma Physics", North-Holland, Amsterdam, 1967. 